Proc. 7th International IEEE Conference on E-Commerce Technology (CEC'05), Munich, Germany, July 2005, pp. 75-82

\title{
A Novel Auction Mechanism for Selling Time-Sensitive E-Services
}

\author{
Juong-Sik Lee and Boleslaw K. Szymanski \\ Optimaret Inc. and Department of Computer Science \\ Rensselaer Polytechnic Institute \\ $1108^{\text {th }}$ Street, Troy, NY 12180, USA \\ \{leej6,szymansk\}@cs.rpi.edu
}

\begin{abstract}
Many e-services are time-sensitive as the users request them for a specific time period. Such services need to be repeatedly offered to keep them constantly utilized. This paper studies winner selection strategies in a recurring auction for such time-sensitive e-services. We observe that because of uneven wealth distribution, the least wealthy bidders tend to drop out of recurring auction as they persistently loose. The bidders dropping out of an auction decrease competition and can cause a collapse of winning prices. We propose and evaluate a novel auction mechanism that enables bidder drop control. Compared to traditional auction mechanisms, ours increases revenue of the e-service provider and decreases loss of fairness of the e-service allocation.
\end{abstract}

\section{Introduction}

E-service is a modular, nimble, Internet-based service that most often requires various time sensitive system resources such as bandwidth, computational power or memory to guarantee the Quality of Service (QoS) to accomplish their tasks. Thus, e-services often have time-sensitive property ${ }^{1}$. Such e-services, if not used for a certain time period, perish, as they cannot be stored in the warehouse for a future use. Moreover, time-sensitive e-services have to be offered for sale repeatedly to keep them being utilized all the time.

Increasing dominance of service-oriented paradigm in the overall U.S. economy underscores the importance of e-services and its important aspect, efficient pricing. In many existing electronic markets, fixed pricing or static time-differential pricing mechanisms are widely used because of their simplicity. However, there is a natural variation in customer's demand over time. For this reason, those pricing mechanisms are insufficient. They lead to under-utilization of time-sensitive

\footnotetext{
${ }^{1}$ Examples of this type of e-services are various Grid Computing Services, including utility computing, and Internet based multimedia services such as Video On Demand (VOD), Video Conferencing, Music on Demand, and so on.
}

resources when demand is low and under-pricing when demand is high. In a static time differential pricing mechanism two or more tiers of on/off peak rates can improve efficiency by partial matching of lower demand with lower price. However, this mechanism still remains inflexible, since demands of customers do not follow a step function, but they shift rather gradually from on- to off-peaks.

A continuously adjustable dynamic pricing mechanism that adapts to changing market conditions is more efficient. It can maximize both time-sensitive resource utilization and revenue in a variety of market conditions. During the low utilization period, the low price invoked by the adaptive pricing can increase competition. During the high demand period, emerging high prices increase the service provider revenue. Moreover, with such a mechanism, the price itself becomes an important signal for controlling fair allocation of resources. However, this very dynamism of pricing makes the service provider's pricing decisions difficult. An auction mechanism can mitigate this difficulty. In electronic market environments, use of an auction provides the several benefits. (1) Auctions are inherently easy to understand by both customers and the service provider. (2) Rules and procedures that define an auction are usually easy to implement in automated electronic environments [3]. (3) An auction eliminates any need for defining complex dynamic pricing structures. (4) Auctions support decentralized pricing and therefore avoid abusive market practices [6].

An auction for time sensitive e-services is in fact a recurring auction, as the traded e-services must be repeatedly resold for future time periods. Applying traditional auction mechanisms, such as English or Vickery ones [7], to such an auction may result in an inevitable starvation for resources for certain customers. As a result, the affected customers may decide to drop out of the future auction rounds, thereby decreasing the long-term demand for e-services. The 
lowered demand may lead to the collapse of the value of the bids needed to win an auction round. In such a development, the e-service provider cannot guarantee the revenues above its minimum cost. To stabilize revenue, the e-service provider must prevent the price collapse and that requires mechanisms to control the resource supply and the bidder drop problem.

In this paper, we propose a novel auction mechanism, called the Optimal Recurring Auction (ORA), for the repeatedly requested time-sensitive e-services. This mechanism focuses on reducing the bidder drop problem, eliminating the resource waste and avoiding an asymmetry of the negotiation power from which the traditional auction mechanisms suffer. The proposed mechanism is applicable to a multiple winner, discriminatory pricing, and sealed bid auction with the seller reservation price. The mechanism attempts to minimize the communication overhead and to maximize the seller's revenue.

The reminder of this paper is organized as follows. In the next section, we briefly describe previous approaches to maximizing the seller's revenue in an auction mechanism design, analyze potential market environments for e-services and define the problems that motivate our work. Section 3 illustrates the novel auction mechanism ORA. The proposed mechanism is verified by various experiments whose results are given in Section 4. Finally, in Section 5, summaries of the contributions and future works are included

\section{Related Works}

\subsection{Seller's Optimal Auction Mechanisms}

The two main approaches proposed previously to maximize the seller's revenue are based on controlling the resource supply and named the guaranteed market clearing price approach and the guaranteed revenue approach. They are often referred to as seller's optimal auction mechanisms [3]. In the guaranteed market clearing price approach, only the bidders bidding higher than the reservation price of the auctioneer (or seller) are winners [4]. In this case, the auctioneer may sell only a part of the entire stock, if this is what is needed to service the winners. In the guaranteed revenue approach, if the revenue that emerges from the winners' bidding dose not meet the expectations of the auctioneer, the entire auction is canceled [5]. This means that in each auction round the auctioneer either sells its entire stock or none at all. In this paper, we refer to those mechanisms as Reservation Price Auction (RPA) and Cancelable Auction (CA), respectively.

\subsection{Potential Market Environments}

Different market structures require different pricing and negotiation mechanism [3]. Hence, precise analysis of market environments is one of the essential steps in the design of an efficient auction-based dynamic pricing and negotiation mechanism.

We assume that there are many customers and one eservice provider in the market. Multiple units of premium-quality, homogeneous e-services are traded. Those e-services require certain amount of predefined time-sensitive resources to guarantee the service premium quality. The customers request repeatedly the desired e-services for a certain (each time different) time interval. After the requested time period ends, the allocated time-sensitive resource becomes free and need to be offered to customers again. Hence, an auction in such a market is a recurring one.

Each customer's true valuation (i.e., her willingness to pay) is restricted by her wealth that is unevenly distributed among customers. We make the following assumptions: the true valuation of each customer is independently distributed (so-called an Independent Private Value Model assumption); each customer keeps his true valuation constant; customers are risk neutral and symmetric. From the assumption of a risk neutral customer and perfectly sealed bids, it follows that each customer $i$ bids the price $b_{i}$ that maximizes his expected payoff (i.e., utility), $U_{i}\left(b_{i}\right)$ defined as

$$
U_{i}\left(b_{i}\right)=\left(t_{i}-b_{i}\right) \cdot q_{i}\left(b_{i}\right),
$$

where $t_{i}$ denotes true valuation of customer $i$ and $q_{i}\left(b_{i}\right)$ denotes the probability of winning with the bidding price $b_{i}$. There is a trade-off relationship between the profit factor (i.e., $t_{i}-b_{i}$ ) and the winning probability, $q_{i}\left(b_{i}\right)$. If the customer bids more, the winning probability rises, but the resulting profit factor decreases. Conversely, if the customer bids less, the profit factor increases at the expense of the winning probability. Likewise, in a recurring auction, if a customer lost in the last auction round, she may increase her bid in the current round to increase the winning probability. If a customer won in the last round, he may maintain the bidding price or decrease it in the current round to increase the profit factor.

\subsection{Motivations for Novel Auction Mechanism}

The traditional auctions, including the seller's optimal auctions, when applied to the time-sensitive e-services, cause the following three problems. 
1) Asymmetric balance of negotiating power: In most of the traditional auction mechanisms, the prices bid in an auction are dependent only on the customer's willingness to pay for the traded goods. This means that intentions of only customers, but not the e-service provider, are reflected in the auction winning prices.

2) Resource waste: To restore the symmetric balance of negotiating power, the seller's optimal auction mechanisms such as RPA and CA, were proposed [4, 5]. However, when the time-sensitive resources are traded, the seller's optimal auctions cause resource waste. In RPA, the reservation price restricts the number of winners. In $\mathrm{CA}$, the cancellation of an auction round wastes the entire stock of resources. Our experimental results presented in Figure 1 show the extent of the resource waste in RPA and CA. $28.6 \%$ of resources in RPA and $23.5 \%$ of resources in CPA are wasted in the market environments of the experiments described in Section 4.1.
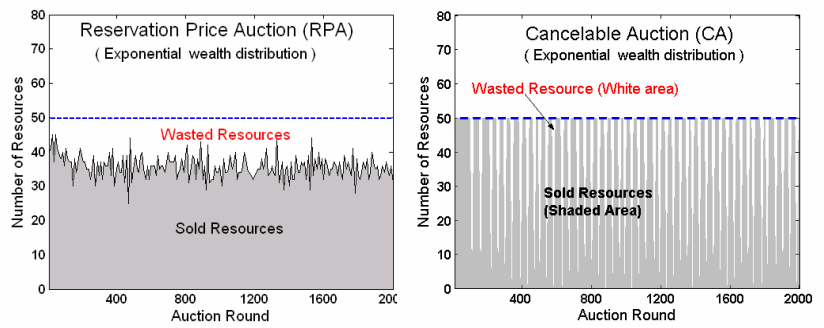

Figure 1. The Resource Waste Problem

3) Bidder drop problem: Prices bid in an auction are dependent on the willingness of each customer to pay. This willingness in turn can be expressed as the customer true valuation. Each customer's wealth influences the upper bound on the customer's willingness to pay. An uneven wealth distribution can cause starvation of poor customers in a recurring auction. A frequent starvation for the traded resources (i.e., e-services in many e-markets) decreases the customer's interest in the future auction rounds. In such a situation, if some customers conclude that it is impossible or unlikely that they will win at the price that they are willing to pay, they will drop from the future auction rounds.

In a recurring auction, each customer's drop out of an auction decreases the number of active customers. We will refer to active customers as "bidders". Such a drop in the number of bidders gradually decreases the price competition. In the long run, when the number of bidders drops below a certain level, the seller can not guarantee the expected revenues in the future auction rounds. This is because the remaining bidders constantly win and as a result they may decrease their bidding prices for future auction rounds to maximize their expected profit. Thus, in such a scenario, the bidding price guaranteeing the win in an auction round may collapse to a very low level. The importance of bidder drop control (i.e., maintaining price competition $)^{2}$ is proved theoretically in our recent studies $[9,10]$. The Figure 2 shows the direct effect of the bidder drop problem on the revenue of the eservice provider based on the experimental scenarios described in Section 4.1.

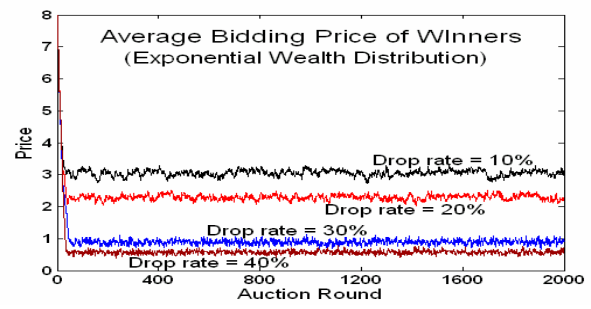

Figure 2. The Bidder Drop Problem

\section{Optimal Recurring Auction Mechanism}

The bidder drop problem in a recurring auction for the time-sensitive e-services is the main reason of the revenue instability resulting from the price collapse. To prevent such instability, we propose an Optimal Recurring Auction (ORA) mechanism that focuses on reducing the bidder drop problem, eliminating the waste of resources, and restoring symmetry of the negotiating power.

The main idea of the mechanism is based on the demand-supply principle of micro-economics [1]. As shown in Figure 3, when demand falls from D1 to D2 during a recurring auction, the minimum marketclearing price decreases from $\mathrm{p} 1$ to $\mathrm{p} 2$. In this case, to maintain or increase the minimum market-clearing price to p1, seller (i.e, auctioneer) must decrease the supply of resources from q1 to q2 (i.e., the entire supply curve needs to change from S1 to S2). Conversely, when the overall bid price increases, the seller may increase the supply. When selling e-service, however, if the seller decreases the supply for a given time period, the unsold resources are wasted. In contrast, in the proposed mechanism, the "unsold" resources (q1 - q2 in Figure 3) are sold to the bidders who have high probability of dropping out of the forthcoming auction round. This solution reduces the

\footnotetext{
${ }^{2}$ In a Vickrey auction, theoretically, the optimal bidding price is independent on the number of participants. Yet, Vickery noticed in his work [7] that when the competition is weak, the revenue may become small. Several other examples of similar phenomena were found in the real world situations reported in [8]. Hence, the bidder drop control is also an important factor in Vickrey auction.
} 
bidder drop problem and keeps enough bidders interested in an auction to maintain the competition for resources. Simultaneously, using "unsold" resources for bidder drop control resolves the resource waste problem.

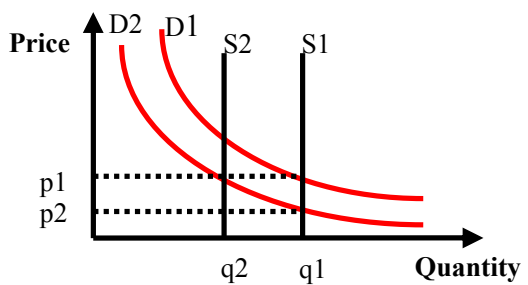

Figure 3. The Demand-Supply Principle

To describe the proposed ORA mechanism based on the above main idea, we define here the fundamental notions of a bidder, bidding price, and goods.

Bidder: There are $n+1$ bidders, denoted by $i=0, \ldots, n$, including $n$ customers, $i=1 . . n$, and the eservice provider $i=0$. Each bidder reports his bidding price $b_{0}, b_{1}, b_{2}, \ldots, b_{n}$ in each auction round. We assume the sealed bidding, thus only a bidder and the e-service provider can communicate.

Goods: There are $R$ units of time-sensitive resources that can be assigned to the homogeneous e-service for the predefined time period. The e-service provider trades these assignments in each auction round. Each bidder requires one unit of time-sensitive resources for the desired guaranteed premium-quality e-service. Thus, in this paper, we regard trading $R$ units of timesensitive resources as trading $R$ units of homogeneous premium quality time-sensitive e-services.

\subsection{Bidder's Class Definition}

The first step of the ORA mechanism is to define the bidders classes based on each bidder's bidding price $b_{i}$, where $i=1, \ldots, n$ and the e-service provider's reservation price $b_{0}$. The e-service provider classifies the bidders into the Definitely Winner (DW), Winner or Loser (WL) and Definitely Loser (DL) classes using the following conditions:

$$
\begin{array}{ll}
i \in D W & \text { if } b_{i} \geq b_{0} \& r_{i}>n-R, \quad i=1,2, \ldots, n, \\
i \in D L & \text { if } b_{i} \leq 0, \quad i=1,2, \ldots, n, \\
i \in W L & \text { otherwise, }
\end{array}
$$

where $r_{i}$ denote rank of bidder $i$ in the increasing order of bidding prices of all customers. In each auction round, the DW class bidders become winners without any additional considerations, since they bid higher than the reservation price of the e-service provider and there are enough resources (i.e., e-service units) to assign one to all of them. The DL class consists of customers who already dropped out of the auction.

The bidders who are in the WL class can be winners or losers depending on the bidder drop control mechanism applied. The numbers of bidders in the DW, WL and DL classes are denoted as $N_{d w}, N_{w l}$ and $N_{d l}$, respectively. Figure 4 shows the bidder's classes in the ORA and compares them with the classes in the traditional auction mechanisms.

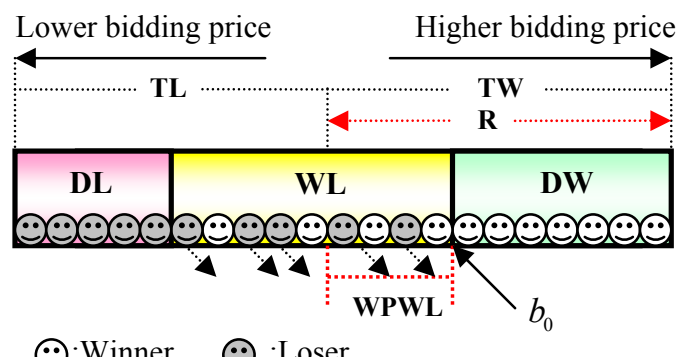

Figure 4. The Bidder's classes in the ORA

In Figure 4, TL and TW denote the Traditional Losers and Traditional Winner classes as defined by the traditional auction mechanism. WPWL represents the Winning Portion of the WL class, and the number of winners in the WL class is denoted by $N_{\text {wpwl }}$. Hence,

$$
N_{w p w l}=R-N_{d w}
$$

The e-service provider's bidding price plays the same role as the reservation price of RPA. By introducing reservation price, the ORA mechanism creates symmetry in balance of the negotiating power from lack of which the traditional auction mechanisms suffer.

\subsection{Bidder Drop Control Mechanism}

By selecting winners in the WL class, the ORA mechanism encourages them to stay in the auction, so the winners should include those bidders in the WL class who are considering dropping out of the auction. Thus, the important role of the bidder drop control is to select winners in the WL class efficiently.

As the first approximation of such a mechanism, we propose the Valuable Last Loser First Bidder Drop Control (VLLF-BDC) algorithm. The main idea behind this algorithm is to allocate the desired e-service to a customer before she drops out of an auction. The algorithm consists of two phases. In the first phase, the 
bidders who lost in the last auction round but bid in the current round higher price than in the previous one are marked as potential winners. The marked bidders are ranked according to their bidding prices and up to the $N_{w p w l}$ highest ranked marked bidders are selected as winners of the current auction round. If the number of the marked bidders is smaller than $N_{w p w l}$, the remaining resources are allocated in the second phase of the algorithm.

The winner selection in the first phase is influenced by the bids and winning record in the previous auction round, so there could be some loss of fairness. To compensate for it, in the second phase, the highest bidding unmarked bidders in the WL class are selected as winners of the remaining resources. By marking only those last losers who bid higher in the current round than in the previous one, the algorithm prevents bidders with low bidding patterns from becoming winners.

\subsection{Optimal Distribution of Resources}

By using a bidder drop control algorithm, the proposed ORA mechanism can maintain price competition and therefore stabilize the e-service provider revenue during the recurring auction. For these purpose, there should be resources reserved for the bidder drop control because allocating all resources to the DW class would reduce the ORA to the traditional auction with all its disadvantages. Hence, for the bidder drop control to work efficiently, the e-service provider must use the optimal reservation price. After all, this is the reservation price of e-service that defines the membership in the DW class.

\subsubsection{Optimal Range of Reservation Price}

Throughout this paper, we define $C_{m}$ as the minimum cost of a unit of traded resources. The e-service provider should set this cost after considering internal and external expenses. This cost can also be interpreted as the e-service provider's desired minimum price for the unit of time-sensitive resources. The specific mechanism for deciding $C_{m}$ is beyond the scope of this paper.

The minimum revenue of an auction round with the bidder drop control should be larger than the e-service provider's profitability revenue. A sufficient condition to ensure this constraint is

$$
b_{0} \cdot N_{d w}+P_{w l m p} \cdot\left(R-N_{d w}\right)>C_{m} \cdot R,
$$

where $P_{w l m p}$ represents the average bidding price of winners in the WL class. Hence

$$
P_{w l m p}>\frac{C_{m} \cdot R-b_{0} \cdot N_{d w}}{R-N_{d w}}
$$

To control bidder drop efficiently, $N_{d w}$ should be smaller than $R$ to preserve some units of time-sensitive resources for the bidder drop control. Hence, the following conditions on $N_{d w}$, and $P_{w l m p}$ can be derived:

$$
0 \leq N_{d w}<R ; \quad 0<P_{w l m p}<b_{0}
$$

Using inequalities (4) and (6), the condition for the optimal reservation price values is

$$
b_{0}>C_{m}
$$

Therefore, the e-service provider should bid a higher price than the minimum cost of a unit of time-sensitive resources to maintain profitability of each auction round.

The upper bound of a range for the optimal reservation price is constrained by the interrelationship between three types of customer's classes and fairness. As shown in Table 1, an increase in the reservation price decreases the number of customers in the DW class (i.e., $N_{d w}$ decreases). This change results in an increase in the number of resource units reserved for the bidder drop control. Thus, in this case, the size of the DL class decreases (i.e., $N_{d l}$ decreases). Since decreasing $N_{d l}$ means increasing price competitions, the resulting total revenue of e-service provider in the recurring auction usually increases (this increase is the largest when $N_{d w}=R$ and then it steadily shrinks and may become negative for larger reservation prices, when $N_{d w}$ is smaller). However, increasing the number of the resource units reserved for the bidder drop control decreases fairness of resource allocation. This is because the winners in the first phase of the VLLF algorithm are selected based not only on their current bidding prices but also on their bidding prices and status in the previous auction round.

\begin{tabular}{|c|c|c|c|c|c|}
\hline $\begin{array}{l}\text { Reserv. } \\
\text { Price }\end{array}$ & $N_{d w}$ & $N_{w p w l}$ & $N_{d l}$ & Revenue & Fairness \\
\hline \hline$\uparrow$ & $\downarrow$ & $\uparrow$ & $\downarrow$ & $\uparrow$ for $N_{d w} \sim \mathrm{R}$ & $\downarrow$ \\
\hline$\downarrow$ & $\uparrow$ & $\downarrow$ & $\uparrow$ & $\downarrow$ for $N_{d w} \sim \mathrm{R}$ & $\uparrow$ \\
\hline
\end{tabular}

$\uparrow:$ Increase $\downarrow$ : Decrease $\sim$ : Close to

Table 1. The interrelationship of customer's classes

The reverse case (i.e., decreasing the reservation price) increases fairness by decreasing the number of units available for the bidder drop control, and may either 
increase or decrease the total revenue of the e-service provider. Accordingly, in deciding the upper bound of the reservation price, the e-service provider should balance an increase in the total revenues versus the loss of fairness induced by the selected reservation price. As shown in Figure 5, based on many experiments conducted under the various customer wealth distributions, we discerned $2 / 3$ rule (i.e., every two out of three time-sensitive resources should be allocated to the DW class) for nearly optimal distribution of the time-sensitive resources between the DW class and the pool of resources reserved for the bidder drop control algorithm. Allocation of $60 \%$ to 70 $\%$ of time-sensitive resources to the DW class in each auction round can achieve the e-service provider's desired revenue and minimize the loss of fairness. This rule leads to a simple and adaptive formula for the eservice provider optimal bidding price $b_{0}$. It should be set to the maximum of the two values: the $2 R / 3$-th highest bid in the current auction round and $C_{m}$, the minimum cost of a unit of the traded resources.

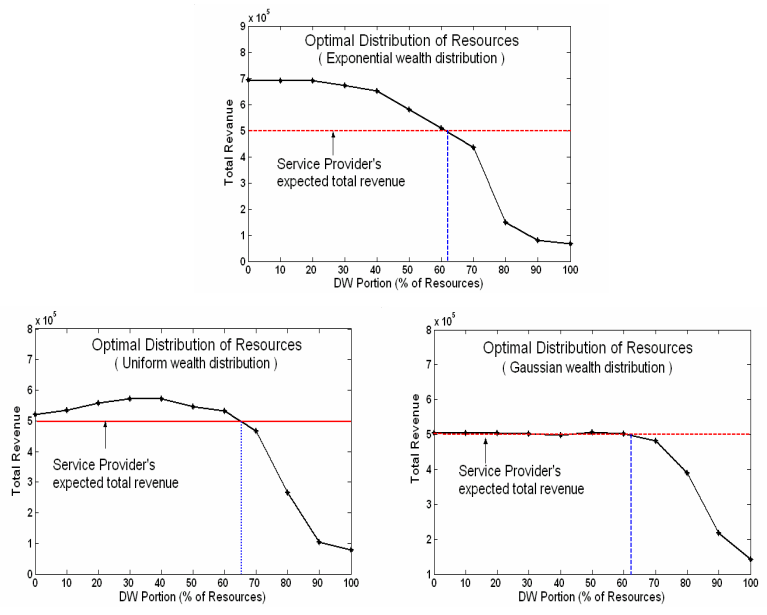

Figure 5. The Optimal Distribution of Resources

\section{Discussion of Experimental Results}

\subsection{Experimentation Scenarios}

In our experimentation, auctions are executed 2000 times recurrently and the following scenarios are simulated.

\subsubsection{Auction Mechanism}

We compare the following five auction mechanisms with multiple winners, one time sealed bid and discriminatory pricing for selling single item homogeneous time-sensitive e-service recurrently.

(1) Traditional Auction (TA) denotes an auction mechanism that has no bidder drop control mechanism.
In TA, bidders drop out of the recurring auction as a result of starvation.

(2) Traditional Auction with No Bidder Drop Assumption (TANBDA) represents a traditional auction mechanism in which bidders never drop during the recurring auction in spite of starvation (i.e., despite the consecutive losses in the recurring auction).

(3) Reservation Price Auction (RPA) represents the TA with reservation price.

(4) Cancelable Auction (CA) denotes auction mechanism in which the e-service provider cancels an auction round when the projected revenue does not meet her expectation.

(5) Optimal Recurring Auction (ORA) represents an auction mechanism that supports the VLLF-BDC algorithm described in the previous section.

The experimental results of TANBDA are impossible to achieve in the real recurring auction, because the no bidder drop assumption is unrealistic. In the real world, starvation, triggered by the uneven wealth distribution, will cause bidders drop. Thus, in our experimentation, TANBDA is only used for comparison.

\subsubsection{Wealth distribution and minimum cost of unit of time-sensitive resources}

The wealth of each customer limits her willingness to pay (i.e., her unit of resources valuation) in the auction. For this reason, we can interpret the wealth distribution as a distribution of the upper bound on willingness to pay. We set the e-service provider's minimum cost of a unit of the resources (e-services) at 5. Thus, we set the reservation price of RPA as 5, too. We consider three types of the standard distributions of the upper bound on willingness to pay among the customers, all with the mean of 5: (1) the exponential distribution, (2) the uniform distribution over [0, 10] range, and (3) the Gaussian distribution.

\subsubsection{Bidders, Bidding Behavior and Goods}

There are 100 customers in our experiments. Initially, all are active, i.e., all are bidders. We assume that the initial bidding price is randomly selected from the range $\left[t_{i} / 2, t_{i}\right]$, where $t_{i}$ represents the upper bound on customer $i$ willingness to pay. The sealed bidding assumption makes each bidder's bidding behavior independent of others. However, in a recurring auction, the bidding behavior is influenced by the results of the previous auction rounds, i.e., the win/loss decision informed to each bidder. Based on the assumption of risk neutral bidders, each bidder will maximize its expected profit. All the above considerations motivated us to assume the following 
bidding behavior. If a bidder lost in the last auction round, she increases her bidding price by a factor of $\alpha>1$ to improve her win probability in the current round. The increase of bidding price is limited by the upper bound on bidder's willingness to pay. If a bidder won in the last auction round, she, with equal probability of 0.5 , either decreases the bidding price by a factor $\beta$ or maintains it unchanged. The decrease attempts to maximize the expected profit. $\alpha$ and $\beta$ are set in the experiments to 1.2 and 0.8 , respectively. The minimum bidding price of a bidder is 0.1 . If a bidder drops out of an auction, his bidding price is set to 0 . There are 50 units of resources (i.e. time-sensitive e-service units) available for allocation in each auction round.

\subsubsection{Tolerance of Consecutive Losses}

The customer's tolerance of consecutive losses, abbreviated as TCL, denotes the maximum number of consecutive losses that a customer can tolerate before dropping out of an auction. TCL of each customer is uniformly distributed over the range of $[2,10]$.

\subsection{Analysis of Results}

Our experiments focus on revenues of the e-service provider and on fairness of resource allocation. An auction is entirely fair if a bidder with a bid higher than a winner is a winner as well. In our experiments, the e-service provider's revenue is proportional to the average bidding price of winners in each auction round, so we use the latter as a measure of the former. We also measure the number of wins for each customer in 2000 rounds of the recurring auction. The resulting distribution is a metric of fairness, because higher bidding customers should be more frequent winners than the lower bidding ones.

Fairness of TANBDA is optimal, because a bidder with the bid higher than a winner is also a winner. Additionally, by the no bidder drop assumption, TANBDA never looses a customer with high willingness to pay and low TCL. This means that TANBDA prevents the loss of fairness that may result from low TCL. Thus, we can measure the loss of fairness of TA, RPA, CA and ORA by their degree of deviation from the fairness of TANBDA. We measure loss of fairness $L F_{k}$ of the auction mechanism $k$ by the distribution of wins between the customers:

$$
L F_{k}=\frac{\sum_{i=1}^{n}\left|N W_{\text {TANBDA }}(i)-N W_{k}(i)\right|}{R \cdot N_{\text {Total_Auction }}} \cdot 100,
$$

where $n$ denotes the total number of customers in the recurring auction, $N W_{T A N B D A}(i)$ and $N W_{k}(i)$ represent the total number of wins by bidder $i$ during $N_{\text {Total } \_ \text {Auction }}$ of auction rounds in TANBDA and auction mechanism $k$, respectively.

In TA, an inevitable bidder drop problem is the dominating factor that decreases the e-service provider's revenue, because there are no wasted timesensitive resources. As described earlier, the bidder drop problem results in a plunge of the average bidding price of auction winners in the long run.

In RPA, the revenue of e-service provider is mainly decreased by the resource waste problem. The effect of bidder drop problem on revenue is small in this case, because the reservation price prevents the winners from decreasing their bidding price to the very low level. However, RPA does not avoid the resource waste problem. As a result, the e-service provider cannot achieve her desired revenue in the recurring auction of this type.

CA suffers from the same problem as RPA. By canceling auction, $\mathrm{CA}$ can prevent remaining customers from decreasing their bidding price to the very low levels. However, the resources wasted in the cancelled auction will prevent the e-service provider from achieving the desired revenue in CA.

ORA is able to maintain price competition permanently in a recurring auction thanks to the efficient bidder drop control. Moreover, in ORA, the resource waste problem never arises, because the entire stock of time-sensitive resources is sold in each auction round. Therefore, the e-service provider can preserve nearly optimal level of the revenue.

As shown in Figure 6, the bidder drop and resource waste problems arise under all simulated wealth distributions in our experimental scenarios.

The loss of fairness of ORA is remarkably lower than the one observed in TA, RPA and CA under all simulated wealth distributions of customers. This phenomenon results from the fact that TA, RPA and CA cannot prevent the loss of fairness caused by high true value bidders dropping out of an auction as a result of exceeding their TCLs. In other words, TA, RPA and CA cannot prevent a customer who is willing to pay high prices but has low TCL from dropping out of an auction after exceeding his TCL at some auction round. In each auction round, TA, RPA and CA have highest possible fairness, because their winners are selected by the current bidding price only. Yet, 
remarkably, ORA has lower loss of fairness over the entire recurring auction because loss of fairness that results from TCL is the dominating factor in the long run. The specific results measuring the loss of fairness under various customer wealth distributions are provided in Table 2 .
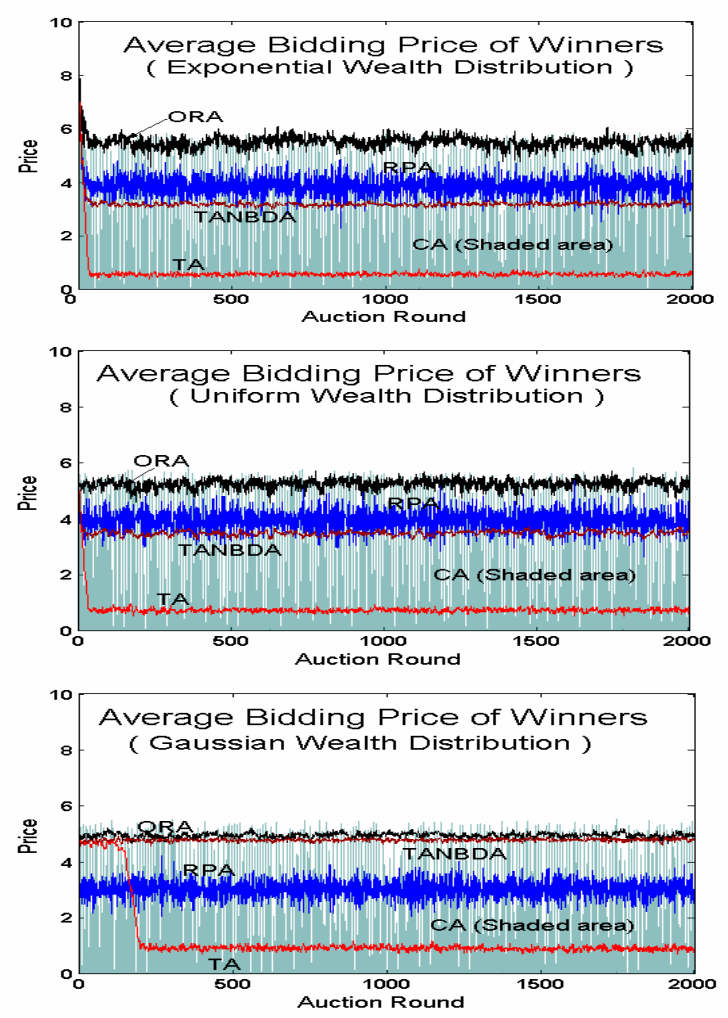

Figure 6. Average winning price of winners

\begin{tabular}{|c||c|c|c|}
\hline & Exponential & Uniform & Gaussian \\
\hline \hline TA & $34.6 \%$ & $23.9 \%$ & $29.4 \%$ \\
\hline CA & $33.5 \%$ & $32.9 \%$ & $33.4 \%$ \\
\hline RPA & $30.0 \%$ & $28.9 \%$ & $41.8 \%$ \\
\hline ORA & $9.4 \%$ & $6.0 \%$ & $11.9 \%$ \\
\hline
\end{tabular}

Table 2. Loss of Fairness

We also simulated the more general case of an auction in which a bidder who dropped out can return when the winning price becomes sufficiently low. For this case, the experimental results show that the revenue of the e-service provider settles somewhere between the revenues of TA and TANBDA because those are the border cases of the general one. The revenue of the TA case sets the lower bound for the revenues in the general case because there are no bidders returning during the recurring auction. The revenue of TANBDA sets the upper bound because all bidders return immediately to the recurring auction in that case. In summary, ORA can achieve the increased revenue and the decreased loss of fairness in the recurring auction for time-sensitive e-services by resolving the bidder drop problem and the resource waste problem.

\section{Conclusions and Future Works}

Since auction mechanism is a competition based dynamic pricing mechanism, the bidder drop problem is one of the most important factors in designing auctioneer's strategy in the recurring auction. Resolving this problem for time-sensitive e-services stabilizes revenue of e-service provider by preventing the collapse of price competitions. Another problem that should be considered for maximizing e-service provider's revenue is the resource waste. The traditional auction mechanisms, including seller's optimal auctions, do not address these problems. The proposed ORA mechanism stabilizes and increases the revenue of the e-service provider by resolving the two problems mentioned above. This mechanism also decreases the loss of fairness by preventing the drop of bidders who have high willingness to pay during the recurring auction. In future works, we plan to study more efficient bidder drop control algorithms. We also plan to investigate a more general case of recurring auction in which heterogeneous multiple unit of timesensitive e-services are traded. Finally, we will also study the theoretical underpinnings of the proposed auction mechanism.

\section{REFERENCES}

[1] R. H. Frank and B.S. Bernanke, "Principle of Economics: $2^{\text {nd }}$ edition", McGraw Hill, New York, 2004.

[2] A. Tiwana and B. Ramesh, "e-Service: Problems, Opportunities, and Digital Platforms", in proceeding of the $34^{\text {th }}$ Hawaii International Conference on System Science, 2001.

[3] M. Bichler, "The Future of e-Markets: Multidimensional Market Mechanism", Cambridge University Press, 2001.

[4] J.G.Riley and W.F.Samuelson, "Optimal Auction", The American Economic Review, Vol. 71, No. 3, Jun. 1981.

[5] A. Fiat and A.V. Goldgerg, J.D. Hartline, and A.R. Karlin, "Competitive Generalized Auction", in Proceeding of $34^{\text {th }}$ Annual ACM symposium on Theory of computing, 2002.

[6] R. McAfee and P.J. McMillan, "Auction and Bidding”, Journal of Economic Literature, Vol. 25, 1997, pp. 699 - 738.

[7]W. Vickrey, "Counter speculation, Auction, and Competitive Sealed Tenders", Journal of Finance, Vol. 16, No. 1, March 1961.

[8]L. Ausubel and P. Cramton, "Vickrey Auctions with Reserve Pricing", Economic Theory, 23, April 2004, pp 493 - 505.

[9] J.S. Lee and B.K. Szymanski, "An Analysis and Simulation of a Novel Auction-Based Pricing Mechanism for Network Services", Technical Report TR-05-02, Department of Computer Science, Rensselaer Polytechnic Institute, Jan. 2005.

[10] J.S. Lee and B.K. Szymanski, "Stabilizing Market via a Novel Auction based Pricing Mechanism for Short-Term Contracts for Network Services", 9 ${ }^{\text {th }}$ IFIP/IEEE International Symposium on Integrated Network Management (IM2005), Nice, France, 2005.

[11] V. Krishna, "Auction Theory", Academic Press, 2002. 